\title{
Un équilibre instable entre le quantitatif et le qualitatif
}

L'enseignement supérieur en Tunisie

An unstable balance between quantity and quality. Higher Education in Tunisia Un equilibrio inestable entre lo cuantitativo y lo cualitativo. La enseñanza superior en Túnez

\section{Riadh Zghal}

\section{CpenEdition}

\section{Journals}

Édition électronique

URL : http://journals.openedition.org/ries/197

DOI : 10.4000/ries. 197

ISSN : 2261-4265

Éditeur

Centre international d'études pédagogiques

Édition imprimée

Date de publication : 1 septembre 2007

Pagination : 51-62

ISBN : 978-2-85420-570-1

ISSN : 1254-4590

Référence électronique

Riadh Zghal, « Un équilibre instable entre le quantitatif et le qualitatif », Revue internationale d'éducation de Sèvres [En ligne], 45 | septembre 2007, mis en ligne le 22 juin 2011, consulté le 30 avril 2019. URL: http://journals.openedition.org/ries/197; DOI : 10.4000/ries.197 


\title{
Un équilibre instable entre le quantitatif et le qualitatif
}

\section{L'enseignement supérieur en Tunisie}

\author{
Riadh Zghal
}

Il est difficile aujourd'hui d'analyser un système d'enseignement supérieur national sans une mise en perspective avec le sens qu'il représente pour la société et son rapport avec son histoire, ni avec les problématiques dont il est l'objet à l'échelle internationale dans un contexte marqué par la globalisation en marche. Dans le cas de la Tunisie, la première université a vu le jour dès le VIII $^{\mathrm{e}}$ siècle à une époque où enseignement religieux et enseignement scientifique étaient indissociés. La colonisation française et les choix adoptés après l'indépendance ont marqué de manière indélébile l'enseignement supérieur actuel qui est caractérisé par un biculturalisme, des liens avec le passé historique et, en même temps, une ouverture sur la modernité ainsi qu'une volonté de convergence avec les standards internationaux dans le domaine. Cela implique de nombreux défis à relever, dont particulièrement des changements de stratégies et des virages politiques difficiles à négocier. En effet, l'enseignement supérieur tunisien a toujours été influencé par les mutations politiques culturelles et sociales qu'a connues le pays.

\section{LE CONTEXTE HISTORIQUE}

La première université tunisienne est celle de la Zitouna, fondée en l'an 734. Elle était hébergée dans la mosquée du même nom, dans un contexte où religion et science étaient indissociées. La Zitouna était un lieu de culte, de savoir et un centre politico-économique où se traitaient maintes transactions. Outre les enseignements littéraires et religieux, on y formait notamment à la philosophie, les mathématiques, la médecine et l'astrologie. D'illustres imams et savants y ont été formés dont Ibn Khaldoun (XIV siècle), auteur de la Mukaddima (les Prolégomènes) et l'un des fondateurs de la sociologie moderne ${ }^{1}$. L'université de la Zitouna supervisait l'enseignement tunisien, en dehors de l'école publique française, tous cycles confondus. Seul l'enseignement supérieur était dispensé à la mosquée, les autres cycles étant installés dans des locaux dispersés dans la médina de Tunis puis, plus tard, dans d'autres médinas des grandes villes du pays. Durant la période coloniale et avec l'avènement de l'école

1. Voir : http://www.uz.rnu.tn, consulté en mai 2007. 
française avec ses deux formules, soit entièrement francophone soit francoarabe, un vent de modernisation a soufflé sur la Zitouna. L'élite a souhaité changer les conditions de formation et de vie des élèves, mais aussi introduire davantage de matières scientifiques dans les programmes d'enseignement.

Le concept d'une éducation s'appuyant à la fois sur la référence à l'héritage civilisationnel arabo-musulman et sur l'ouverture à la modernité est né à l'université de la Zitouna. Il a donné naissance par la suite à l'enseignement sadikien totalement bilingue. Ce modèle dit «sadiki» en référence à la medersa Essadikia ${ }^{2}$ est celui qui, en Tunisie, a inspiré les choix politiques de l'éducation depuis l'indépendance jusqu'à nos jours.

Bourguiba, leader de la lutte pour l'indépendance et premier président de la république tunisienne, avait une approche à la fois laïque et jacobine. Sous prétexte d'unifier l'enseignement national, il avait mis fin à la vocation universitaire de la Zitouna. Plus tard, en 1987, le président Ben Ali qui lui a succédé, crée - ou «ressuscite»- l'Université Ez Zitouna, cette fois hors de la mosquée. Ce sera une université moderne qui répond aux mêmes critères d'organisation et de gestion que les autres universités du pays et qui se spécialisera non seulement dans les sciences religieuses (islam et autres religions), mais également dans les langues anciennes et les civilisations qui ont nourri la civilisation islamique.

Il nous a semblé important de rappeler cet héritage pour une meilleure compréhension du système de l'enseignement supérieur tunisien actuel et de ses problématiques. Toutefois, nous ne perdons pas de vue le fait que ces dernières reflètent, en partie du moins, celles qui se posent à travers le monde.

\section{UN CONTEXTE \\ DE GLOBALISATION CROISSANTE}

L'enseignement supérieur a fait l'objet de nombreuses rencontres et de débats, particulièrement à la fin des années 1990, et souvent à l'initiative d'instances internationales à la recherche de définitions de nouvelles orientations adaptées aux nouvelles conditions d'un monde en mutation.

Ainsi, au printemps 1998, l'université de Pékin a abrité, à l'occasion de son centenaire, un forum international auquel ont participé 94 représentants et présidents de 92 universités parmi les plus renommées dans le monde. Selon nous, une convergence de points de vue s'est dégagée autour de plusieurs points ${ }^{3}$ :

- avec l'émergence d'une économie globale basée sur le savoir, l'université prend une plus grande importance dans le monde. «En tant que moteur du développement social, l'université a contribué énormément à la transmission, au traitement, à la dissémination, à l'application et à la création du savoir»;

2. Fondée en 1875, la medersa (collège) par le bey Mohammed es Sadok, dont elle porte le nom.

3. Chen Xiangming (1998) «Opportunities and Challenges of the University: Presidents Meet at Peking University», International Higher Education, N. 13, automne 1998, p. 18. 
- l'université a glissé de la périphérie vers le centre de la société, occupant désormais une place stratégique;

- face aux nouvelles opportunités et aux nouveaux défis de l'ère de l'information, l'université est appelée à développer de nouvelles stratégies d'enseignement et d'apprentissage, en introduisant les nouvelles technologies comme outil pédagogique;

- les étudiants doivent être encouragés à étudier de façon indépendante avec l'aide d'Internet et des équipements multimédia. Mais les formateurs devraient aussi leur fournir des opportunités pour l'interaction sociale qui est vitale, compte tenu des développements futurs des valeurs humanitaires et de l'éthique;

- pour tout cela, l'université est appelée à tracer les nouvelles lignes de sa structure interne de sorte que sa gestion devienne plus efficiente et plus efficace et que la relation entre la recherche et l'enseignement soit équilibrée de façon adéquate;

- pour survivre et prospérer, l'université devra fournir des efforts particuliers en vue de préserver son héritage culturel tout en promouvant l'internationalisation de la formation supérieure à travers le monde ${ }^{4}$.

Au cours de l'automne de la même année, l'UNESCO organisa une conférence mondiale sur l'enseignement supérieur précédée de conférences régionales organisées de 1996 à 1998. La déclaration finale de cette conférence définit les missions de l'enseignement supérieur, dont l'éducation, la formation, la recherche, la contribution au développement durable et l'amélioration de la société en tant que tout. Elle insiste également sur certaines valeurs qui devraient être présentes dans les institutions universitaires, à savoir : l'éthique, la rigueur intellectuelle, l'esprit critique, la vision à long terme. Pour assurer la référence à de telles valeurs, on rappelle que le personnel enseignant et les étudiants devraient bénéficier d'une entière autonomie académique et de liberté tout en étant responsables et «responsables» (accountable) devant la société. Étant au centre du processus de développement, l'enseignement supérieur devrait aussi bénéficier de la contribution de tous les acteurs sociaux et institutionnels.

Dans un rapport au titre suggestif, Higher Education in Developing Countries, Peril and Promise, publié en 2000, la Banque mondiale prône une approche fondée sur la contribution de l'enseignement supérieur au développement économique, social et politique. Cette contribution devrait être appréciée sous un angle historique, comparatif et prospectif prenant en compte les défis de l'avenir ainsi que la participation des différentes parties prenantes.

On remarque aisément la convergence de vision entre dirigeants universitaires et instances internationales concernant les principes directeurs du futur de l'enseignement supérieur. Les contours d'un référentiel international sont

4. Ibid. 
ainsi balisés : l'université est désormais reconnue comme un acteur stratégique du développement, les principes d'autonomie et d'indépendance des enseignants et des étudiants, d'éthique, de participation des différentes parties prenantes dans les réformes, d'internationalisation et d'exploitation des technologies disponibles pour l'enseignement sont soulignés.

Mettre en application ces principes revêt une véritable transformation de l'université classique. En vue d'assumer un nouveau rôle désormais central pour l'insertion dans une économie globalisée et mue par le savoir et l'innovation, il faudra gérer des ruptures avec l'ordre établi et traiter de nouvelles problématiques. Parmi les plus récurrentes, il y a celle de l'expansion de l'enseignement supérieur à des couches de plus en plus larges de la population en âge de fréquenter l'université. Lorsque la croissance économique crée une demande en cadres et en qualifications supplémentaires et que l'augmentation du revenu des familles stimule la motivation pour la formation supérieure et continue, le secteur public, pourvoyeur historique de cette formation, devient incapable de répondre à la demande. Il s'ensuit une opportunité offerte au développement de l'enseignement supérieur privé, soutenue par l'orientation vers une économie libérale ce qui introduit de nouvelles incertitudes ${ }^{5}$. Au Portugal par exemple, l'explosion de la demande pour l'enseignement supérieur s'est produite dans les années 1980. Dans les années 1990, la stabilisation de la demande conjuguée avec l'expansion des établissements publics a entraîné la mise en difficulté des institutions privées dont certaines ont fini par arrêter leur activité. La vulnérabilité de ces dernières a été renforcée par leur concentration sur des filières populaires et bon marché ainsi que par la concentration géographique qui a favorisé une rude concurrence sur un espace délimité et un nombre réduit de spécialités ${ }^{6}$. Cet exemple illustre le type de problématique lié à une politique de développement de l'investissement privé non encadrée par une stratégie à long terme. Le contre exemple est celui du Japon et de la Corée du Sud où plus de $80 \%$ des étudiants sont inscrits dans des institutions privées non subventionnées par l'État. ${ }^{7}$

Il existe un autre défi auquel se heurtent la plupart des systèmes d'enseignement supérieur, corollaire de l'énorme expansion des effectifs d'étudiants : celui de la qualité des diplômes. La qualité apparaît comme une question cruciale, vu son impact important à la fois sur les perspectives d'emploi des diplômés, sur l'intégration des économies nationales dans une économie mondialisée et sur l'attraction des investissements directs étrangers. Outre les choix politiques nationaux en matière d'organisation des institutions universitaires

5. Altbach P. G. (2001) «The Rise of the Pseudouniversities», International Higher Education, $\mathrm{n}^{\circ} 25$, automne 2001.

6. Teixeira P. N. (2006) «When Expansion Ends», International Higher Education, ${ }^{\circ}$ 44, pp. 14-15.

7. Altbach P. G. (2006) «The Achille heel of India's High-Tech Future: World-Class Universities », International Higher Education, $\mathrm{n}^{\circ} 44$, pp. 16-17. 
et des cursus, la qualité de la formation est aussi tributaire de la concurrence à l'échelle nationale et internationale. Cette dernière détermine la capacité des universités d'attirer les enseignants les plus qualifiés et les étudiants les plus brillants.

Chaque pays a besoin d'une élite formée de telle sorte qu'elle puisse exercer dans une économie globalisée et répondre à ses exigences. Parallèlement, chaque pays a besoin d'offrir une formation universitaire accessible aux catégories sociales les moins favorisées. Ce double objectif représente un autre défi lancé à l'enseignement supérieur aujourd'hui. Servir ces deux objectifs en dotant le pays d'une élite, tout en veillant à respecter les principes d'équité sociale en offrant les mêmes opportunités à tous, est un pari difficile à tenir. Le risque pour les universités, si l'on fait prévaloir le principe d'un enseignement universitaire pour le plus grand nombre, est de s'embourber dans une baisse incontrôlée de la qualité de la formation. Les meilleurs étudiants, issus des couches sociales les plus favorisées, ne trouvant pas l'offre de formation convenant à leurs attentes, s'orienteront vers les universités étrangères exerçant dans ou hors du pays. En revanche, les très bons étudiants issus des classes sociales moins favorisées ne disposent pas des moyens pour financer une formation de qualité et peuvent être considérés comme une perte de capital humain, à moins que des opportunités exceptionnelles ne s'ouvrent à eux. Dans ces conditions, parler d'équité devient impertinent, car c'est plutôt le contraire qui s'est produit : l'égalitarisme a éliminé les opportunités dont auraient pu bénéficier les plus doués. La formation d'une élite peut se réaliser par le biais d'institutions universitaires d'excellence. C'est pourquoi l'analyse comparative et l'évaluation de la qualité sont désormais les éléments constitutifs de la stratégie visant à tirer les institutions universitaires vers le haut ${ }^{8}$. À cet égard, on peut citer l'exemple de l'Allemagne où le ministre Bulmahn a lancé en 2004 «l'initiative pour l'excellence » destinée à mettre en compétition les institutions universitaires qui souhaitent bénéficier de fonds spéciaux destinés à renforcer leurs capacités de formation et de recherche ${ }^{9}$.

Les systèmes d'accréditation des institutions universitaires et des diplômes, initiés d'abord par les États-Unis puis diffusés à travers le monde, reflètent cette recherche de qualité de l'enseignement supérieur. Le besoin d'assurance de la qualité dans le fonctionnement des institutions universitaires est motivé également par la concurrence entre ces dernières ainsi que par celle qui se joue, à l'échelle internationale, en matière d'attraction des étudiants migrants. C'est que le secteur de l'enseignement supérieur est aussi considéré dans son aspect économique de création de la valeur.

8. Cf. le Processus de Bologne adopté par les pays de l'Union Européenne.

9. Kehm B. M. (2006) «The German "Initiative for Excellence" and Rankings », International Higher Education, $\mathrm{n}^{\circ} 44$, pp. 20-22. 


\section{LA STRATÉgIE D'INTÉGRATION DE LA TUNISIE DANS UN CONTEXTE MONDIAL}

Il ne fait pas de doute que la Tunisie est concernée par ces problématiques et ces défis auxquels s'expose l'enseignement supérieur actuellement dans le monde. Néanmoins, les politiques actuelles ne peuvent être saisies dans leur sens profond sans référence à la société, à son histoire et plus précisément celle qui concerne l'éducation et les choix civilisationnels.

À cet égard, on peut retenir l'attrait qu'ont toujours exercé sur l'élite tunisienne, et de façon concomitante, le savoir et l'ouverture sur le monde et sur la modernité. Aujourd'hui, la Tunisie tente de s'intégrer positivement dans un contexte international "globalisé », ce qui a des implications déterminantes sur l'éducation et l'économie nationales. L'insertion dans le système économique mondial signifie le développement d'une économie du savoir où la création de la valeur s'appuie principalement sur la maîtrise des nouvelles technologies, la recherche scientifique, l'innovation et les activités de service. Cela induit des exigences précises pour l'enseignement supérieur et aussi la formation professionnelle. La maîtrise de la technologie et l'innovation exigent un système de formation qui, au-delà de la transmission du savoir, développe des habiletés pratiques, des capacités intellectuelles mais aussi psychiques pour exercer la créativité et accepter la prise de risque. Elles nécessitent également des capacités morales pour assumer ses responsabilités, supporter l'échec avec résilience, travailler en équipe et chercher à créer des synergies par la communication et la coopération avec des personnes venant d'autres horizons. Tout cela s'oppose au modèle traditionnel de l'enseignement orienté vers le savoir théorique spécialisé favorable à la formation d'une "pensée tubulaire ${ }^{10}$ » qui prédispose à une appréhension partielle de la réalité et rend aveugle à sa complexité.

À cet égard, le cloisonnement entre les niveaux de formation, du moins au niveau des paradigmes fondateurs, est improductif voire dangereux. La connexion entre un système de formation, un système de recherche et un système de production a du mal à se réaliser alors qu'elle représente une condition pour le développement de l'économie du savoir. Néanmoins, l'État accorde des financements croissants à la recherche scientifique qui devraient atteindre 1,25\% du PIB en 2009. Le concept d'enseignement supérieur licence-mastère-doctorat, qui inclut une composante professionnelle, a été adopté et sa mise en place est en cours. La présence de modules techniques jusque dans les cursus littéraires, les mastères professionnels, l'explosion des formations spécialisées pour mieux

10. Le concept vient de Roskis E. (1978) dans son ouvrage How real is real? Communication Desinformation, Contusion, N.Y. Basic Books inc., cité par Lapointe J. "Stratégie des organisations apprenantes et nouveaux modes d'acquisition du savoir » in Nouveaux modes d'acquisition du savoir et travail humain (1999), Essid Y. (dir), Faculté des lettres et sciences humaines et Faculté des sciences économiques et de gestion de Sfax, Tunisie. 
répondre à la demande d'un marché du travail tiré par l'innovation et la technologie, l'appel à la contribution des professionnels aux enseignements universitaires, tout cela est inscrit dans l'agenda de la réforme de l'enseignement supérieur. Mais la mise en application sera d'autant plus problématique que les rigidités et les inerties ont longtemps été renforcées par une gestion bureaucratique et centralisée, ceci au niveau institutionnel ${ }^{11}$.

$\mathrm{Au}$ niveau des représentations sociales, les rigidités viennent d'un investissement collectif intense dans un secteur considéré comme le principal ascenseur social par les classes moyennes et celles les moins favorisées, et comme une garantie de maintien dans une position sociale avantageuse par les classes favorisées. De telles attitudes ont conduit progressivement à une valorisation si démesurée des diplômes qu'elle a fait perdre de vue l'importance du savoirfaire, du savoir communiquer et du savoir être pour une insertion dans la vie active. Un problème majeur se pose actuellement à l'enseignement supérieur tunisien : celui de l'opposition entre les représentations sociales dominantes relatives à son utilité et le rôle moteur qu'il doit jouer pour le développement, grâce à la maîtrise des sciences et de la technologie, l'exercice des capacités créatives et à l'innovation. Les premières donnent au diplôme une valeur en soi, les secondes exigent de réelles capacités intellectuelles de savoir et de savoir-faire en plus des capacités psychologiques qui prédisposent à la création, la prise de risque, la recherche de synergies à travers le travail en équipe et le respect d'une certaine déontologie. Les représentations sociales pèsent lourdement sur les choix politiques et favorisent la levée des barrières à l'entrée de l'université ainsi que, en amont, une baisse en cascade des résultats exigés pour le passage d'un niveau à l'autre du système éducatif.

\section{L'EXPANSION DE L'ENSEIGNEMENT SUPÉRIEUR ET LA STRATÉGIE NATIONALE}

Le secteur universitaire public tunisien est actuellement composé de treize universités, vingt-quatre instituts supérieurs des études technologiques et trois instituts de formation des maîtres. Au total, le secteur public compte cent quatre-vingt-dix institutions universitaires. Le secteur privé, relativement récent, ne compte que seize institutions appelées tantôt université tantôt institut ou faculté. Elles sont généralement de petite taille et n'attirent qu'une proportion infime d'étudiants (4 200 en 2006 dont seulement 1500 Tunisiens) ${ }^{12}$.

\footnotetext{
11. Voir Zouari-Bouattour S. (1998) «L'enseignement supérieur en Tunisie : les enjeux» in Plassard J. M. et Ben Sedrine S. (dir.) Enseignement supérieur et insertion professionnelle en Tunisie, Université des sciences sociales de Toulouse, pp. 50-71.

12. Sur l'enseignement supérieur et la recherche scientifique en Tunisie, voir : www.bawaba.gov.tn/, le portail de l'administration tunisienne. Voir aussi les données de base de l'enseignement supérieur sur : www.mes.tn/ (consultés en mai 2007).
} 
La formation délivrée par ces structures est concentrée sur les disciplines de management, d'informatique appliquée à la gestion et, dans une moindre mesure, d'ingénierie informatique ${ }^{13}$. Le secteur privé, qui bénéficie d'incitations à l'investissement particulièrement généreuses parmi lesquelles l'acquisition du terrain pour un dinar symbolique, n'a pas encore réussi à décoller. De ce fait, le poids de l'expansion de l'enseignement supérieur pèse presque totalement sur le secteur public, ce qui est d'ailleurs cohérent avec les choix politiques $\mathrm{du}$ pays qui a toujours misé sur l'éducation comme principal moteur du développement.

Le budget de l'enseignement supérieur a représenté 5,27\% du budget de l'État en 2006, celui de l'éducation nationale et de la formation professionnelle 16,46\%. Les deux premières décennies qui ont suivi l'indépendance (1956) ont été employées à généraliser l'accessibilité des enfants aux cycles primaire et secondaire de l'enseignement dans tout le pays. L'enseignement universitaire n'était assuré que dans la capitale. Un système de bourses permettait à ceux qui obtenaient les meilleurs résultats au baccalauréat de poursuivre, s'ils le souhaitaient, des études supérieures à l'étranger dans une large palette de pays.

Dès le début des années 1970, pour répondre à une forte demande sociale d'enseignement supérieur motivée, entre autres, par des taux élevés de réussite au baccalauréat a été enclenché un processus de création d'institutions universitaires puis de véritables universités dans les régions.

Au plan politique, l'enseignement supérieur est présenté comme l'une des priorités du programme électoral du Président de la république et l'un des piliers de la stratégie de développement du pays. La stratégie relative à ce secteur converge avec les orientations des réformes dans le monde évoquées plus haut. Elle inclut dans ses objectifs l'internationalisation, l'introduction des nouvelles technologies et la réforme pédagogique. Cette stratégie est également motivée par la nécessité de parer au chômage des diplômés, problème qui s'amplifie depuis une décennie. Elle met l'accent, entre autres, sur la multiplication des filières en vue de renforcer l'employabilité des diplômés, l'accroissement de la proportion des diplômés dans les sciences de l'ingénieur (passer de six pour mille actuellement à onze pour mille ${ }^{14}$ ) afin de développer les capacités nationales d'innovation technologique, et enfin la co-diplômation en coopération avec des universités étrangères. Ces orientations visent in fine une convergence de l'enseignement supérieur tunisien avec celui des pays de l'Union européenne, d'une part, et le développement d'un capital humain disposant de capacités adaptées aux exigences de la nouvelle économie du savoir et de la globalisation, d'autre part.

13. Sur les mastères professionnels délivrés par les établissements privés de l'enseignement supérieur, voir : www.mes.tn/ (consulté en mai 2007).

14. Interview du ministre de l'enseignement supérieur, avril 2007. 
Dans un discours prononcé en juin $2006^{15}$ devant les dirigeants des institutions universitaires, le ministre de l'enseignement supérieur a présenté la nouvelle stratégie de l'enseignement supérieur. Elle s'oriente à servir les objectifs économiques stratégiques et s’inscrit dans une approche qualité, comme il apparaît dans les éléments du discours suivants :

- intégration de l'économie nationale dans une économie mondialisée tirée par le savoir et les technologies de l'information et de la communication;

- renforcement du partenariat entre le secteur de l'enseignement supérieur et de la recherche scientifique et l'environnement social et économique; ce partenariat devrait aboutir à des profils de diplômés à la fois adaptés aux besoins de l'économie et attractifs pour les investissements directs étrangers, favoriser un apport d'expertise scientifique et de recherche et développement aux entreprises; la formation devrait aussi stimuler l'esprit d'entreprise notamment pour la réalisation de projets innovants par les diplômés eux-mêmes;

- adoption par le système tunisien du modèle international dit LMD dont la mise en place a démarré en septembre 2006 et se terminera en septembre 2008; une note diffusée sur le site du ministère définit ce qui est attendu de ce système, entre autres : la mobilité nationale et internationale des étudiants, la flexibilité, la comparabilité internationale et la facilité d'insertion professionnelle.

Sous la contrainte de la quantité, la politique qui était dirigée essentiellement par le souci d'offrir une place à l'université à chaque détenteur du baccalauréat, semble virer vers un souci de qualité, sans toutefois abandonner le principe du droit de tous les bacheliers à l'enseignement supérieur. Il s'agit à la fois de satisfaire le besoin du pays de disposer d'une élite formée, et d'offrir une formation universitaire accessible à tous. Cela représente évidemment un défi particulièrement difficile à relever dans les conditions actuelles. La qualité de la formation du supérieur a en effet beaucoup souffert, ces dernières décennies, du fait d'effectifs pléthoriques et du sous encadrement. L'accroissement vertigineux des effectifs continue sans interruption depuis une quarantaine d'années : de 13000 en 1975-76, ils sont passés à 137000 en 1997-98. En douze ans, les effectifs ont été multipliés par dix, et l'on prévoyait une stabilisation à 300000 étudiants en 2010. Mais ils atteignent aujourd'hui le nombre de 340392 . On prévoit qu'ils continueront à augmenter jusqu'à atteindre 500000 en 2009. Selon les prévisions du ministère, le sommet de la courbe sera atteint en 2014, année charnière à partir de laquelle se déclencherait la tendance à la décroissance.

Il est évident que la capacité du système à former les formateurs n'a pas évolué au même rythme que celui des effectifs étudiants. En effet, les universités fonctionnent actuellement avec une forte proportion de contractuels, d'assistants titulaires de mastères et de professeurs de l'enseignement secondaire titulaires

15. www.mes.tn/discours_ministre_juin2006.pdf/, consulté mai 2007. 
de la maîtrise et sans formation dans la recherche scientifique ${ }^{16}$. La part des professionnels participant à la formation est encore beaucoup trop réduite pour être en mesure d'alimenter un enseignement professionnalisé.

\section{LES DÉFIS À RELEVER}

L'adoption d'une politique orientée vers la qualité représente en réalité une rupture avec la situation actuelle. Le projet qualité annoncé par le ministère concerne les différentes composantes du système de l'enseignement supérieur (scientifique, pédagogique, organisationnelle). Il implique un véritable changement de paradigme dans le fonctionnement du système universitaire et le comportement des acteurs.

Au plan pédagogique, les changements de méthode concerneront et les enseignants et les étudiants. La focalisation de l'attention de ces derniers sur l'obtention du diplôme d'une part, et celle de l'administration sur la «rentabilité interne» des institutions mesurée par les taux de réussite aux examens, d'autre part, ont gravement nui à la qualité des diplômes, notamment ceux à forte composante théorique et non assortis de stages pratiques et de projets de fin d'études.

Le souci des notes a favorisé le "par cœur», la passivité en classe, l'absence de curiosité, d'esprit critique et de synthèse ${ }^{17}$. Il a également réduit l'engouement pour la lecture et l'envie d'apprendre par soi-même.

La méthode pédagogique axée sur les cours magistraux plaçant l'enseignant dans la position de source principale du savoir, un savoir à restituer lors des épreuves d'examen, enlève à l'enseignement sa pertinence. En effet c'est dans la mesure où les connaissances sont présentées dans un rapport cohérent d'échange entre formateur et apprenant qu'elles sont utilisables dans la vie concrète et qu'elles suscitent l'intérêt de l'apprenant ${ }^{18}$. L'idée que «l'enseignement le plus précieux que les élèves retirent d'un cours n'est pas la maîtrise d'une discipline, mais la prédisposition à apprendre ${ }^{19}$ est aujourd'hui largement partagée par les spécialistes de la pédagogie universitaire. Dans un environnement en pleine mutation, la formation devient un processus qui dure toute la vie. En conséquence, la pédagogie appliquée dans les universités devra muter vers des méthodes interactives et motivantes pour des étudiants appelés à prendre en charge leur propre formation à court et long terme.

16. Selon le ministère, l'enseignement supérieur public fonctionne actuellement avec un corps enseignant composé à $60 \%$ d'assistants (non titulaires d'une thèse de doctorat) et maîtres-assistants (titulaires d'un doctorat), à $12 \%$ de contractuels (titulaires d'un mastère) et à seulement $12 \%$ de professeurs, le reste étant composé de vacataires, professeurs de l'enseignement secondaire (en particulier pour l'enseignement ciblé des langues) et de professeurs invités. 17. Zghal R. (1999) «Formation et défis de la globalisation : employé formé ou organisation qualifiante » in Essid Y. (dir) Nouveaux modes d'acquisition du savoir et travail humain, Faculté des lettres et des sciences humaines de Sfax et Faculté des sciences économiques et de gestion de Sfax, pp. 59-66.

18. Elmore R. F. (1994) «Avant-propos» in C.R. Christensen, D.A. Garvin, A. Sweet, Former à une pensée autonome, De Boek Université, Nouveaux Horizons.

19. Ibid., pp. xii-xiii. 
Au plan organisationnel et en l'absence d'autonomie des universités, les réformes sont conduites par le ministère selon une logique d'uniformisation des enseignements et de nivellement des institutions. Les cursus déterminés pour chaque maîtrise s'appliquent à toutes les institutions universitaires de même spécialité; les grandes lignes du contenu de chaque module sont définis à l'échelle nationale en ce qui concerne les troncs communs; à l'intérieur d'une même institution, les enseignements sont coordonnés par un enseignant de la spécialité et il est demandé que l'épreuve de l'examen soit la même pour toutes les classes de même niveau.

Si une telle conception vise à assurer une formation de base pour des étudiants dispersés dans des institutions inégalement fournies en ressources humaines, et à mettre fin aux chasses gardées de certains enseignants puissants et conservateurs, elle introduit de nouvelles rigidités lorsqu'il s'agit d'adapter les enseignements à l'évolution de la science. Or les changements rapides que connaissent la science et l'environnement économique et social exigent plutôt une plus grande flexibilité dans le fonctionnement des institutions universitaires et l'élaboration des cursus universitaires.

Le fonctionnement actuel des universités est dominé par la logique administrative : cloisonnement des institutions et absence de mobilité des étudiants pour suivre les programmes spécialisés là où ils sont offerts, absence de cursus réalisés en coopération entre institutions, quasi absence de programmes de recherche en coopération inter-institutions... Finalement, l'université a une existence plus administrative que pédagogique et scientifique. Cela est en contradiction avec le contexte international où l'enjeu réside dans la coopération en réseau, la mise en commun des moyens pour affronter la concurrence à tous les niveaux et dans tous les domaines. Des changements profonds au niveau organisationnels sont donc à réaliser pour réussir la stratégie annoncée et s'aligner sur les référentiels internationaux qui sont déjà assez clairement définis.

Enfin l'un des principaux défis à relever, qui apparaît en filigrane dans le chapitre du XI ${ }^{\mathrm{e}}$ plan 2007-2011 réservé à l'enseignement supérieur, est l'employabilité des diplômés. La stratégie adoptée jusque-là se base sur la diversification des spécialités et le renforcement de celles qui sont jugées "prometteuses", autrement dit liées à l'économie du savoir ${ }^{20}$. Mais l'incertitude de l'environnement appelle une révision continue de la palette des spécialités programmées. Le ministère prévoit la mise en place d'un observatoire au niveau de chaque université pour assurer une veille stratégique quant à la demande du marché. L'efficacité d'un tel instrument dépendra naturellement de la réactivité des institutions universitaires et donc de leur autonomie effective en matière de gestion du corps enseignant, de leur capacité d'attirer les professionnels pour

20. 1284 spécialités figurent actuellement dans les programmes des différentes universités; l'objectif annoncé par le plan est d'élever la proportion des spécialités liées à l'économie du savoir à $32 \%$ de l'ensemble des spécialités offertes. 
une contribution à la formation, d'amélioration de la qualité de la formation et de développement de relations d'échange avec l'environnement.

L'employabilité des diplômés est aussi liée aux attitudes des agents économiques et de la société en général vis-à-vis de la science et des techniques. Leur bienveillance vis-à-vis du savoir diffusé par les universités sera d'autant plus importante que l'apport de ces dernières sera palpable au niveau de la recherche et développement $(R \& D)$ et de la solution des problèmes rencontrés dans la vie des organisations et celle des individus. Il est donc demandé à l'université de se positionner par rapport aux besoins de la société et de prospecter les changements de la structure du marché de l'emploi ${ }^{21}$. C'est la référence aux sciences, y compris les sciences humaines et sociales, et à la technologie dans les activités humaines qui crée la demande pour les détenteurs d'un savoir scientifique ou technique et non pas leur présence sur le marché. Sans une demande sociale réelle pour le savoir, les diplômés de l'enseignement supérieur seront exposés aussi bien au chômage qu'à l'emploi disqualifié. Éviter un tel dérapage représente aujourd'hui le principal défi à relever pour les stratégies de l'enseignement supérieur.

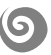

L'enseignement supérieur tunisien bénéficie aujourd'hui d'une reconnaissance politique en tant que secteur stratégique pour le développement économique et social du pays et, par suite, son insertion dans un environnement désormais globalisé. Pour être mise en œuvre avec succès, la stratégie annoncée par les autorités nécessite un changement de paradigmes dans le fonctionnement du système. Si l'objectif ultime est de produire des diplômés compétitifs à une échelle internationale et de faire bénéficier les structures économiques de l'apport de la science, de la technologie et des capacités d'innovation du capital humain formé, il est nécessaire de reconsidérer le sens social donné aux diplômes, de rompre avec l'approche administrative centralisée et cloisonnée de la gestion des institutions et des réformes. Aujourd'hui, la gestion des universités nécessite davantage d'autonomie, de flexibilité pour s'adapter aux besoins du contexte, de créativité, de synergies, d'exploitation optimale des ressources intellectuelles et matérielles et d'émergence de pôles d'excellence qui puissent jouer le rôle de locomotive et de référence pour l'ensemble des institutions. Il s'agit maintenant, pour l'enseignement supérieur tunisien, de réussir le passage d'une stratégie orientée vers l'accueil d'effectifs de plus en plus importants dans les universités, vers une stratégie orientée par la qualité et la compétitivité du capital humain formé, aussi bien à l'échelle nationale qu'à l'échelle internationale.

21. J. Ehrenfeld, P. Conceição, M. Heitor \& P. Vieira (1999). "Towards sustainable universities: challenges for engineering education in the learning economy ", The third International Conference on Technology Policy and Innovation, Austin, 30 août -2 septembre. 